Bull. Austral. Math. Soc.

$34 \mathrm{~K} 15,39 \mathrm{~A} 10,58 \mathrm{~F} 03$

VoL. 57 (1998) [37-48]

\title{
SOLUTION BEHAVIOUR IN A CLASS OF DIFFERENCE-DIFFERENTIAL EQUATIONS
}

\author{
A.D. Fedorenko, V.V. Fedorenko, A.F. Ivanov and A.N. Sharkovsky
}

Difference equations with piecewise continuous nonlinearities and their singular perturbations, first order neutral type delay differential equations with small parameters, are considered. Solutions of the difference equations are shown to be asymptotically periodic with period-adding bifurcations and bifurcations determined by Farey's rule taking place for periods and types of solutions. Solutions of the singularly perturbed delay differential equations are considered and compared with solutions of the difference equations within finite time intervals. The comparison is based on a continuous dependence of solutions on the singular parameter.

\section{INTRODUCTION}

Consider delay differential equations of the form

$$
\mu[\dot{x}(t+1)+c \dot{x}(t)]+x(t+1)=f(x(t))
$$

where $\mu, c$ are constants, $\mu \geqslant 0,|c|<1$, and $f$ is a piecewise continuous function defined by

$$
f(x)= \begin{cases}g(x), & \text { if } \quad 0 \leqslant x \leqslant b, \text { and } \\ h(x), & \text { if } \quad a<x \leqslant 1,\end{cases}
$$

with $a, b$ satisfying $0<a \leqslant b<1$ and $g, h$ are monotonic increasing continuous functions defined on $I=[0,1]$ and such that $0<h(x)<x<g(x)<1$ for all $x \in(0,1)$ (and, hence, $h(0)=0, g(1)=1)$.

When $\mu=0$ the delay differential equation (1) becomes a continuous time difference equation

$$
x(t+1)=f(x(t)) .
$$

Received 13th January, 1997

This work has been supported in part by the International Science Foundation (grant U6G200) and by the State Committee of Ukraine on Science and Technology (grant 1(1).3/81) (A.D.F., V.V.F., and A.N.S.), by the Australian Research Council (A.F.I. and A.N.S.), and by the Isaac Newton Institute for Mathematical Sciences, Cambridge, UK (A.N.S.).

Copyright Clearance Centre, Inc. Serial-fee code: 0004-9729/98 \$A2.00+0.00. 
The qualitative behaviour of solutions of equations (1) and (3) is determined by properties of the map

$$
x \mapsto f(x) .
$$

Equations in the form (1) and (3) appear in many applications. For example, they come from certain boundary value problems for hyperbolic partial differential equations $[1,5,6,7,8,10]$, which describe such real life phenomena as electromagnetic, sound and other types of oscillations. Equation (1), which mathematically is a singular perturbation of equation (3), is a more "accurate" model where viscosity effects are taken into account. There is a number of publications on singularly perturbed delay differential equations, however, most of them deal with the case of the retarded type perturbations corresponding to $c=0$ in (1); see [4] for further references. In this paper we study singularly perturbed equations of the neutral type $(c \neq 0)$, moreover, when the nonlinearity $f$ is a multi-valued function. This type of nonlinearity frequently appears, for example, in control theory [3].

We consider cases $\mu=0$ and $\mu>0$ separately. When $\mu \neq 0$, solutions of equation (1) are studied on finite time intervals. Our approach in this case is based on the continuous dependence of solutions on all involved parameters, in particular, on the continuous dependence on the parameter $\mu$ at $\mu=0$.

If $a<b$ function $f$ is two-valued on the interval $(a, b]$ and, therefore, we have to make additional assumptions in order to obtain a uniquely defined trajectory $x_{n}=f^{n}\left(x_{0}\right), n=$ $0,1,2, \ldots$, for each $x_{0}$. If $x_{0} \in(a, b]$ we set $x_{1}=g\left(x_{0}\right)$; if $x_{n} \in(a, b]$ for $n>0$ we use the following rule (which is the ordinary rule under the existence of "hysteresis"): if $x_{n}=g\left(x_{n-1}\right)$, then $x_{n+1}=g\left(x_{n}\right)$; if $x_{n}=h\left(x_{n-1}\right)$, then $x_{n+1}=h\left(x_{n}\right)$ too. Under such an agreement map, (4) generates a dynamical system with a so-called two-sheeted phase space. For $n \geqslant 0$ it is defined by

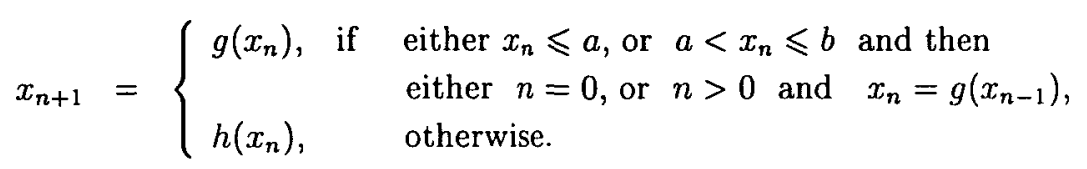

Since $h(a)<f(x) \leqslant g(b)$, for all $x \in I$, it can be assumed that

$$
f(x)= \begin{cases}g(x), & x \in I_{1}, \\ h(x), & x \in I_{2},\end{cases}
$$

where $I_{1}=(h(a), b], \quad I_{2}=(a, g(b))$. If the intervals $I_{1}$ and $I_{2}$ are considered as two different sheets of the phase space then, by identifying the endpoints $a$ and $h(a)$ of the interval $I_{1}$ and the endpoints $b$ and $g(b)$ of the interval $I_{2}$, one gets a circle $S$ made up of two $\operatorname{arcs} I_{1}$ and $I_{2}$. In this case function $f$ defines a generally discontinuous one-to-one map on the circle $S$. In particular, if $g^{-1}(b)$ exists and is less than $b, h^{-1}(a)$ exists and is 
greater than $a$, the function $f$ assumes the following form

$$
f(x)= \begin{cases}g(x), & x \in I_{1} \backslash\left[g^{-1}(b), b\right] \\ h(g(x)), & x \in\left(g^{-1}(b), b\right], \\ h(x), & x \in I_{2} \backslash\left[a, h^{-1}(a)\right], \\ g(h(x)), & x \in\left(a, h^{-1}(a)\right) .\end{cases}
$$

Thus one gets a dynamical system defined on a circle. This naturally leads to "Farey's rule" for the bifurcations of periodic and close to periodic solutions of corresponding difference and differential-difference equations. In the remaining part of the paper, however, we shall not employ this approach of the reduction to a circle map, but will deal instead with the map (4) directly.

In what follows we shall consider map $f$ defined by (5) whose parameters $a$ and $b$ belong to the domain $\Omega=\{\omega=(a, b) \mid 0<a \leqslant b<1\}$. Also we shall always assume that the conditions

$$
g(0)>h(1), \quad \text { that is } g([0, b]) \cap h((a, 1])=\emptyset,
$$

and

$$
g, h \in \operatorname{Lip}_{L}(I, I) \quad \text { with } \quad L<1
$$

are satisfied.

For any measurable set $B \subset I$, mes $f(B) \leqslant L$ mes $B$ and hence mes $f^{n}(B) \rightarrow 0$ as $n \rightarrow 0$. In particular, mes $f^{n}(I) \rightarrow 0$ as $n \rightarrow \infty$ implying mes $A(f)=0$ where $A(f)=\bigcap_{n=0}^{\infty} f^{n}(I)$. This means that the set $A(f)$ is nowhere dense. Therefore, the attractor of the dynamical system (5) is contained inside a nowhere dense set of the interval $I$.

Let $\mathcal{D}(f)=\left\{x \in(0,1) \mid \exists n \geqslant 0, f^{n}(x) \in\{a, b\}\right\}$, that is, $\mathcal{D}(f)$ is a set of points from $(0,1)$, each of which gets mapped into $a$ or $b$ after a finite number of iterations. The set $\mathcal{D}(f)$ can be finite (possibly empty) or infinite. The set $\mathcal{D}(f)$ is a nowhere dense set [9].

The following fundamental property is obvious.

Lemma A. If $J$ is an interval from $I \backslash \mathcal{D}(f)$ then for each $n>0, f^{n}(J)$ is also an interval, and for any two points $x^{\prime}, x^{\prime \prime} \in J,\left|f^{n}\left(x^{\prime}\right)-f^{n}\left(x^{\prime \prime}\right)\right|<L^{n}\left|x^{\prime}-x^{\prime \prime}\right|$.

Thus, the set $\mathcal{D}(f)$ defines a partition of the set $I \backslash \mathcal{D}(f)$ into a finite (if $\mathcal{D}(f)$ is finite) or countable number of intervals $J_{s}, s=1,2, \ldots$, with disjoint interiors which form a Markov partition of the phase space: for any interval $J_{s^{\prime}}$, there exists interval $J_{s^{\prime \prime}}$ such that $f\left(J_{s^{\prime}}\right) \subseteq J_{s^{\prime \prime}}$. It allows us to reduce the study of dynamical system (5) to the analysis of possible paths of the graph induced by the transfer matrix of the Markov partition. In particular, closed loops of the graph correspond to periodic trajectories of the dynamical system. From the analysis of the graph it follows that the statements: 
(i) $\mathcal{D}(f)$ is a finite set,

(ii) there exits an asymptotically periodic trajectory,

(iii) every trajectory is asymptotically periodic

are equivalent $[2,9]$.

Typical for the domain $\Omega$ situation is that every trajectory of the map $f$ is asymptotically periodic $[\mathbf{2}, \mathbf{9}]$ : parameter values for which the map $f$ has asymptotically periodic trajectories form a set containing an open and dense subset of $\Omega$. Almost always the map $f$ has either one or two periodic trajectories. In the latter case the difference between the periods equals 2 .

We shall call any function $\psi: \mathbf{R}^{1} \rightarrow G$, where $G$ is a finite set from $\mathbf{R}^{1}$, a stepfunction.

The following lemma is a consequence of Lemma A.

Lemma B. Assume $\mathcal{D}(f)$ is a finite set. Then there exists an integer $m>1$ such that for any $x \in I$ the limit $\lim _{i \rightarrow \infty} f^{m i}(x)$ exists and the limiting function $f^{*}(x)$ is a stepfunction equal to a constant on each interval of $I \backslash \mathcal{D}(f)$. Moreover, for any $\varepsilon>0$ there are integers $m>0$ and $N>0$ such that $\left|f^{m n}(x)-f^{*}(x)\right|<\varepsilon$ for any $x \in I \backslash \mathcal{D}(f)$ and $n>N$.

\section{Properties of SOlUtions of the Difference EQUATION}

In this section we consider the continuous time difference equation (3). We shall use an integral metric which is given by the norm $\|x\|_{[\alpha, \beta]}:=\int_{\alpha}^{\beta}|x(t)| d t$.

THEOREM 1. If $\mathcal{D}(f)$ is a finite set, then every solution of difference equation (3) with continuous argument converges to a periodic step-function in the integral metric as $t \rightarrow+\infty$.

Proof: The statement follows from the fact that the function $x(t)=f^{n}(\varphi(\{t\})), n=$ $0,1, \ldots$ (here $\{t\}$ is the fractional part of $t$ ), is the solution of the equation $x(t+1)=$ $f(x(t))$ with the initial function $\varphi(t), 0 \leqslant t<1$. If the map $f$ has periodic points, this solution tends in the integral metric to a periodic step-function defined by $x^{*}(t+j)=f^{j} f^{*}(\varphi(t)), j=0,1, \ldots, m-1$, where $f^{*}(x)=\lim _{i \rightarrow \infty} f^{m i}(x), m=n$ or $n(n+2)$, and $n$ is a lesser period of two possible periodic points of the map $f$.

We need a more detailed classification of periodic trajectories based not only on their periods but also using the so-called type of the trajectories $[2,9]$.

Let $P$ be a periodic trajectory of map $f$ for some $\omega^{\prime}=\left(a^{\prime}, b^{\prime}\right) \in \Omega$, and $P_{1}=\{\beta \in$ $P \mid f(\beta)=g(\beta)\}, \quad P_{2}=\{\beta \in P \mid f(\beta)=h(\beta)\}$. Let $\beta_{1}<\beta_{2}<\ldots<\beta_{n_{1}}$ be points of the set $P_{1}$ and $\beta_{n_{1}+1}<\ldots<\beta_{n_{1}+n_{2}}$ be points of the set $P_{2}$. Denote by $p$ the number of points $\beta \in P_{1}$ whose preimages belong to $P_{2}$. Then the number of points $\beta \in P_{2}$ whose preimages belong to $P_{1}$ is also $p$. We shall call $p$ a turn number for the trajectory $P$. 
The trajectory $P$ generates a cyclic permutation $\pi$ of length $n_{1}+n_{2}: \pi(i)=j$ if and only if $f\left(\beta_{i}\right)=\beta_{j}, \quad 1 \leqslant i, j \leqslant n_{1}+n_{2}$.

For every periodic trajectory $P$ of the map (5) there exist positive coprime integers $p, q, p<q$, and a nonnegative integer $d$ such that the cyclic permutation generated by the periodic trajectory $P$ has the form $[2,9]$

$$
\pi(i)= \begin{cases}i+p, & \text { if } \quad 1 \leqslant i \leqslant q-2 p+d \\ i+p+d, & \text { if } q-2 p+d<i \leqslant q-p+d \\ i-q+p-d, & \text { if } q-p+d<i \leqslant q+d \\ i-p, & \text { if } q+d<i \leqslant q+2 d\end{cases}
$$

if $2 p \leqslant q$, and if $2 p>q$, it equals $h \circ \pi \circ h$, where $h(i)=q+2 d+1-i, i=1, \ldots, q+2 d$.

From formula (10) one can see the meaning of $p, q$ and $d$. The period of the trajectory $P$ generating the cyclic permutation $\pi$ is $q+2 d$, and $p$ is the turn number of the trajectory $P$.

In what follows, we always assume that $p$ and $q$ are coprime numbers, $p<q$, and $d$ is a nonnegative integer. We shall call the triple $(p, q, d)$ the type of the periodic trajectory.

Let $\Omega_{p, q, d}=\{\omega \in \Omega \mid$ the periodic trajectory of map (5) has type $(p, q, d)\}$. It follows from $[2,9]$, that for every triple $(p, q, d)$, the sets $\Omega_{p, q, d}$ and int $\Omega_{p, q, d}$ are nonempty connected sets with a rectagle shape. In particular, $\Omega_{p, q, d}^{0}=i n t \Omega_{p, q, d} \backslash\left(\bar{\Omega}_{p, q, d-1} \cup \bar{\Omega}_{p, q, d+1}\right)$ is a nonempty set. If $\omega \in \Omega_{p, q, d}^{0}$, then the map $f$ has a unique periodic trajectory which attracts all other trajectories (here $\Omega_{p, q,-1}=\emptyset$ ).

Suppose the map $f$ has a unique periodic trajectory forming the cycle $\left\{\beta_{1}, \ldots, \beta_{n}\right\}$. If $\pi$ is a cycle permutation generated by this periodic trajectory, then every limit stepfunction $x^{*}$ has the following property: if $x^{*}\left(t_{0}\right)=\beta_{i_{0}}$ at $t_{0} \in \mathbf{R}^{1}$, then $x^{*}\left(t_{0}+j\right)=\beta_{\pi^{j}\left(i_{0}\right)}$ for any positive integer $j$. Thus, in this case, periodic step-functions generated by the equation $x(t+1)=f(x(t))$ can also be classified by cyclic permutations and, hence, by the triple $(p, q, d)$ introduced above. We shall call this triple the type of the periodic step-function, too.

Moreover, it makes a sense to call a triple $(p, q, d)$ the type of the solution of the difference equation (3) if the limiting step-function for this solution is of the type $(p, q, d)$. This allows us to state the following properties of solutions.

Theorem 2. Let $\omega \in \Omega_{p, q, d^{-}}^{0}$. For any function $\varphi:[0,1] \rightarrow[0,1]$, the solution $x_{\varphi}$ of difference equation (3) with initial function $\varphi$ is of type $(p, q, d)$.

The theorem is a direct consequence of Lemma $B$, in accordance with the introduced classification.

The properties of the bifurcation diagram for types of periodic trajectories of the map $f$ were described in $[2,9]$. It was shown that there are two types of bifurcations of periodic trajectories, namely, bifurcations determined by Farey's rule and also the socalled period-adding bifurcations. By making use of Theorem 2, the analogous properties 
of solutions of difference equation (3) can be formulated immediately as follows

THEOREM 3. Let $\omega_{i} \in \Omega_{p_{i}, q_{i}, d_{i}}^{0}, i=1,2$ be two collections of parameters. Then

(Farey's rule) if $p_{1} / q_{1}<p_{2} / q_{2}$, then for any arc $\mathcal{L}$ inside $\Omega$ connecting $\omega_{1}, \omega_{2}$, and any $p, q$ such that $p_{1} / q_{1}<p / q<p_{2} / q_{2}$, there exists $\omega \in \mathcal{L}$ for which the type of solutions of difference equation (3) is $(p, q, d)$ with some $d \geqslant 0$;

(period-adding) if $p_{1} / q_{1}=p_{2} / q_{2}$ and $d_{1}<d_{2}$, then there is an arc $\mathcal{L}$ inside $\Omega$, connecting $\omega_{1}, \omega_{2}$, such that a) for any $\omega \in \mathcal{L}$, the corresponding solution of difference equation (3) has type $\left(p_{1}, q_{1}, d\right)$ with some $d, d_{1} \leqslant d \leqslant d_{2}$, and b) for any $d^{\prime}, d_{1}<d^{\prime}<d_{2}$, there exists $\omega^{\prime} \in \mathcal{L}$ for which type of solutions of equation (3) is $\left(p_{1}, q_{1}, d^{\prime}\right)$.

\section{BeHAVIOUR OF SOLUTIONS OF SINGULARLY PERTURBED EQUATIONS ON FINITE} TIME INTERVALS

In this section, the results on asymptotic behaviour of solutions of difference equation (3) and properties of bifurcation diagrams are translated to the delay differential equation (1). The latter can be considered as a singular perturbation of equation (3) if $\mu$ is sufficiently small. This approach via the singular perturbations allows us to use the continuous dependence of solutions on parameters for the difference-differential equations involved which can be established within finite time intervals.

3.1. Continuous DEPENDENCE OF SOlutions on SINGular Parameter Consider equation (1) again. As it has been shown in [4], in the case $c=0$ the asymptotic behaviour as $t \rightarrow \infty$ of solutions of equations (3) and (1) can be essentially different. Therefore, generally speaking the same differences persist when one compares the more general neutral type differential equation (1) with its limiting case at $\mu=0$, the difference equation (3). In spite of the diferences in the asymptotic behaviour one can expect, however, the closeness between the solutions at least within finite time intervals. Indeed, similar to the case $c=0$, this type of closeness also takes place for equation (1).

Differential equation (1) can be rewritten in the following integral form

$$
\begin{aligned}
x(t+1) & =-c x(t)+(c / \mu) \int_{0}^{t} \exp \{(s-t) / \mu\} x(s) d s \\
& +(1 / \mu) \int_{0}^{t} \exp \{(s-t) / \mu\} f(x(s)) d s \\
& +[c x(0)+x(1)] \exp \{-t / \mu\}, \quad t \geqslant 0
\end{aligned}
$$

allowing us to consider "solutions" to equation (1) which are only piecewise continuous functions.

To construct a solution of equation (1) for $t>1$ one needs an initial function $\varphi$ which can be integrated in the initial interval $[0,1]$ according to the above integral equation. 
Equation (1) is solved then by using the step method. Denote the set of all piecewise Lipschitz continuous functions defined on the interval $[0,1]$ by $P L$. Throughout this section we shall always assume that $\varphi \in P L$. The corresponding solution $x_{\varphi}^{\mu}(t)$ is then a continuous function which is piecewise differentiable for $t>1$. Note that the Lipschitz condition here is a technical one only, which is chosen to match the corresponding assumption on the function $f$.

In contrast with the case $c=0$, there is no invariance property for the solutions of equation (1). Therefore, given an initial function $\varphi \in P L$ the corresponding solution $x_{\varphi}^{\mu}$ may leave the interval $I$ for some $t>1$. This prompts us, in order to be able to compare solutions of equations (1) and (3), to extend the function $f(x)$ beyond the interval $I$ by setting

$$
f(x)=1 \quad \text { if } \quad x>1 \text { and } f(x)=0 \text { if } x<0 .
$$

For any $\varphi \in P L$ the solution $x_{\varphi}^{\mu}(t)$ of equation (1) is a continuous function for $t>1$, while the corresponding solution $x_{\varphi}(t)$ of equation (3) is in general a discontinuous function for $t>1$ with jump discontinuities. The discontinuities of $x_{\varphi}(t)$ come from several different sources. Firstly, they are the discontinuities of the initial function $\varphi$ that are mapped by $f$ into the next time interval. Secondly, one generally has a jump discontinuity at integer points $t_{i}=i, i \in \mathrm{N}$, if the consistency condition for the initial function, $\varphi(1)=f(\varphi(0))$, does not hold. And finally, a jump discontinuity arises every time when the value of the initial function hits the discontinuity of $f$ for that particular sheet which is being used at that moment. This in particular means that there is no uniform closeness between solutions of equations (1) and (3). However they are close in the integral metric.

The following theorem is our principal result about the closeness in the integral metric between solutions of equations (1) and (3) for small $\mu>0$.

THEOREM 4. Suppose $\varphi \in P L$ and let $\varepsilon>0, T>0$ be arbitrary. There exist $\delta>0$ and $\mu_{0}>0$ such that $\left\|x_{\varphi}^{\mu}-x_{\psi}\right\|_{[0, T]}<\varepsilon$ for any $\psi \in P L$ with $\|\varphi-\psi\|_{[0,1]}<\delta$ and every $0<\mu \leqslant \mu_{0}$.

Proof: Let $\varphi \in P L$ and $T>0$ be given and let $0 \leqslant t_{1}<t_{2}<\cdots t_{k} \leqslant T$ be the discontinuity set of the corresponding solution $x_{\varphi}(t)$ in $[0, T]$. Obviously the result can be proved by induction by establishing the closeness on the interval $\left[1, t_{1}\right]$ first, then on the interval $\left[t_{1}, t_{2}\right]$, and so on until the last interval of the continuity set of $x_{\varphi}$. Since a specific sheet of $f$ is used on each of the intervals, the function $f(\varphi(t)), t \in[0, T]$, is piecewise Lipschitz continuous. Therefore, it is sufficient to show the integral closeness within the first interval $\left[1, t_{1}\right]$ assuming that both $\varphi(t)$ and $f(\varphi(t)), t \in\left[0, t_{1}-1\right]$ are Lipschitz continuous functions in there. Due to the same induction argument one can also let $t_{1}=1$. To continue with the proof we need several auxiliary statements.

Lemma C. Suppose $\varphi, \psi \in P L$ are fixed. Then for every $\varepsilon>0$ there exist $\delta>0$ 
and $\mu_{0}>0$ such that $\left\|x_{\varphi}^{\mu}-x_{\psi}^{\mu}\right\|_{[1,2]}<\varepsilon$ for all $0<\mu \leqslant \mu_{0}$ provided $\|\varphi-\psi\|_{[0,1]}<\delta$.

Proof: From the integral equation (11) it follows that the difference $\Delta(t+1):=x_{\varphi}^{\mu}(t+1)-x_{\psi}^{\mu}(t+1), t \in[0,1]$ satisfies the equation

$$
\begin{aligned}
\Delta(t+1) & =-c \Delta(t)+[\Delta(1)+c \Delta(0)] \exp \{-t / \mu\} \\
& +(1 / \mu) \int_{0}^{t} \exp \{(s-t) / \mu\}[f(\varphi(s))-f(\psi(s))] d s \\
& +(c / \mu) \int_{0}^{t} \exp \{(s-t) / \mu\}[\varphi(s)-\psi(s)] d s, \quad t \geqslant 0 .
\end{aligned}
$$

We shall estimate next each component of this equation in the integral metric over the interval $[0,1]$. We have first

$$
\|-c x(t)\|_{[0,1]}=\int_{0}^{1}|-c \Delta(t)| d t=c \int_{0}^{1}|\varphi(t)-\psi(t)| d t \leqslant c \delta .
$$

Since $\varphi(t), \psi(t)$ are fixed and piecewise Lipschitz continuous, there is a constant $M>0$ such that $|\varphi(t)| \leqslant M$ and $|\phi(t)| \leqslant M$, for all $t \in[0,1]$. This gives us

$$
\begin{aligned}
\|[\Delta(1)+c \Delta(0)] \exp \{-t / \mu\}\|_{[0,1]} & =\mid \int_{0}^{1}[\Delta(1)+c \Delta(0)] \exp \{-t / \mu\} d t \\
& \leqslant \delta(1+|c|) \int_{0}^{1} \exp \{-t / \mu\} d t \leqslant \mu \delta(1+|c|) .
\end{aligned}
$$

Next we have

$$
\begin{aligned}
\|(c / \mu) \int_{0}^{t} \exp & \{(s-t) / \mu\}[\varphi(s)-\psi(s)] d s \|_{[0,1]} \\
= & (|c| / \mu) \int_{0}^{1} \int_{0}^{t} \exp \{(s-t) / \mu\}|\varphi(s)-\psi(s)| d s d t \\
& =(|c| / \mu) \int_{0}^{1} \int_{0}^{1} \exp \{(s-t) / \mu\}|\varphi(s)-\psi(s)| d t d s \\
& =|c| \int_{0}^{1}|\varphi(s)-\psi(s)|(1-\exp \{(s-1) / \mu\}) d s \leqslant|c| \cdot \delta .
\end{aligned}
$$

Using the piecewise Lipschitz condition $|f(x)-f(y)| \leqslant K|x-y|$, likewise we can derive the estimate

$$
\left\|(1 / \mu) \int_{0}^{t} \exp \{(s-t) / \mu\}[f(\varphi(s))-f(\psi(s))] d s\right\|_{[0,1]} \leqslant L \cdot \delta .
$$

Now the required estimate on $\|\Delta(t)\|_{[1,2]}$ follows from (12) and (13-16).

Lemma D. Assume $\varphi \in P L$. For arbitrary $\varepsilon>0$ there exists $\mu_{0}>0$ such that $\left\|x_{\varphi}^{\mu}-x_{\varphi}\right\|_{[1,2]}<\varepsilon$ for all $0<\mu \leqslant \mu_{0}$.

Proof: We make use of the fact that

$$
(1 / \mu) \int_{0}^{t} \exp \{(s-t) / \mu\} d s=1-\exp \{-t / \mu\}
$$


Therefore, for any function $a(t)$

$$
a(t)=a(t) \exp \{-t / \mu\}+(1 / \mu) \int_{0}^{t} \exp \{(s-t) / \mu\} a(t) d s .
$$

Since $x_{\varphi}(t+1)=f(\varphi(t)), t \in[0,1]$, and in view of the integral equation (12), one then has for $t \in[0,1]$

$$
\begin{aligned}
x_{\varphi}^{\mu}(t+1)-x_{\varphi}(t+1) & =-c \varphi(t)+(c / \mu) \int_{0}^{t} t \exp \{(s-t) / \mu\} \varphi(s) d s \\
& +(1 / \mu) \int_{0}^{t} \exp \{(s-t) / \mu\} f(\varphi(s)) d s-f(\varphi(t)) \\
& +[\varphi(1)+c \varphi(0)] \exp \{-t / \mu\} \\
& =[\varphi(1)-f(\varphi(t))+c(\varphi(0)-\varphi(t))] \exp \{-t / \mu\} \\
& +(1 / \mu) \int_{0}^{t} \exp \{(s-t) / \mu\}[f(\varphi(s))-f(\varphi(t))] d s \\
& +(c / \mu) \int_{0}^{t} \exp \{(s-t) / \mu\}[\varphi(s)-\varphi(t)] d s .
\end{aligned}
$$

The nonintegral part of the latter expression can be estimated as follows

$$
\begin{aligned}
\|\varphi(1)-f(\varphi(t))+c(\varphi(0)-\varphi(t)) \exp \{-t / \mu\}\|_{[0,1]} & \leqslant 2(M+|c|) \int_{0}^{1} \exp \{-t / \mu\} d t \\
& \leqslant 2(M+|c|) \mu .
\end{aligned}
$$

Using next the Lipschitz property of the initial function, $|\varphi(t)-\varphi(s)| \leqslant L_{1} \cdot|s-t|$, we have

$$
\begin{aligned}
\|(c / \mu) \int_{0}^{t} \exp & \{(s-t) / \mu\}[\varphi(s)-\varphi(t)] d s \|_{[0,1]} \\
& \leqslant(|c| / \mu) \int_{0}^{1} \int_{0}^{t} \exp \{(s-t) / \mu\}|\varphi(s)-\varphi(t)| d s d t \\
& \leqslant \frac{|c| L_{1}}{\mu} \int_{0}^{1} \int_{0}^{t} \exp \{(s-t) / \mu\}|s-t| d s d t \\
& \leqslant \frac{|c| L_{1}}{\mu} \int_{0}^{1}\left(\mu^{2} \int_{0}^{t / \mu} v \exp (-v) d v\right) d t \\
& \leqslant|c| L_{1} \mu .
\end{aligned}
$$

Likewise, using the Lipschitz property of function $f$ with the constant $L$, we can obtain

$$
\left\|(1 / \mu) \int_{0}^{t} \exp \{(s-t) / \mu\}[f(\varphi(s))-f(\varphi(t))] d s\right\|_{[0,1]} \leqslant|c| L_{1} L \mu .
$$

Combining the inequalities (17-19) we therefore have

$$
\left\|x_{\varphi}^{\mu}(t)-x_{\varphi}(t)\right\|_{[1,2]} \leqslant C \cdot \mu \text { for some constant } C,
$$

which immediately implies the statement of the Lemma.

Now to complete the proof of Theorem 4, we apply Lemmas C and D to the inequality

$$
\left\|x_{\psi}^{\mu}-x_{\varphi}\right\| \leqslant\left\|x_{\psi}^{\mu}-x_{\varphi}^{\mu}\right\|+\left\|x_{\varphi}^{\mu}-x_{\varphi}\right\| .
$$




\subsection{FAREY'S RULE For SOlutions OF SINGULARLY PERTURBED DELAY DifFEREN-} TIAL EQUATIONS We shall introduce first several notions allowing us to classify solutions of equation (1) within finite time intervals and to extend Theorems 1 and 2 to the case of small positive $\mu$.

We call a function $z(t), \varepsilon$-periodic with period $s$ on the interval $\left[T_{1}, T_{2}\right]$ with $T_{2}>$ $T_{1}+s$ if $\|z(t)-z(t+s)\|_{\left[T_{1}, T_{2}-s\right]}<\varepsilon\left|T_{2}-T_{1}-s\right|$.

The following lemma is an obvious implication of Lemma B.

LEMMA E. If map (5) has a unique cycle with period $s$ then for any $\varepsilon>0$, there exists $T_{0}$ such that solution $x_{\varphi}$ of equation (3) satisfies

$$
\left\|x_{\varphi}(t)-x_{\varphi}(t+s)\right\|_{[T, T+s]}<\varepsilon s .
$$

for any $T>T_{0}$ and for any initial function $\varphi$.

Thus, in this case, every solution of equation (3) is $\varepsilon$-periodic on any interval $\left[T_{0}, T\right]$ if $T_{0}+s<T<\infty$.

We call a function $z(t), t \in\left[T_{1}, T_{2}\right], \varepsilon$-periodic of the type $(p, q, d)$ if it is $\varepsilon$-periodic with period $s=q+2 d$ on the interval $\left[T_{1}, T_{2}\right]$ and there exists a periodic step-function $z_{0}(t)$ of the type $(p, q, d)$ such that $\left\|z(t)-z_{0}(t)\right\|_{[T, T+s]}<\varepsilon s$ for $T \in\left[T_{1}, T_{2}-s\right]$.

LEMmA F. If the conditions of Lemma $E$ are satisfied and the triple $(p, q, d)$ is the type of the cycle of map (5), then any solution of equation (3) is an $\varepsilon$-periodic function of type $(p, q, d)$ on each interval $\left[T_{1}, T_{2}\right]$ for any $T_{2}>T_{1}+q+2 d$.

It should be noted that the type of solutions of equation (3) is defined uniquely if $\varepsilon$ is sufficiently small.

Due to the continuous dependence of the solutions of equation (3) on the parameter $\omega$ within any of the domains $\Omega_{p, q, d}^{0}$, the following lemma is obviously true.

Lemma G. For any triple $(p, q, d)$, for arbitrary $\varepsilon>0$ and $\delta>0$, there exist a parameter set $\Omega_{p, q, d}^{\prime} \subset \Omega_{p, q, d}^{0}$ with mes $\left(\Omega_{p, q, d}^{0} \backslash \Omega_{p, q, d}^{\prime}\right)<\delta$ and $T>T_{0}+q+2 d$ such that for any $\omega \in \Omega_{p, q, d}^{\prime}$ and for any initial function $\varphi$, the solution $x_{\varphi}$ of the difference equation (3) is an $\varepsilon$-periodic function of type $(p, q, d)$ on the interval $\left[T_{0}, T\right]$.

Now, using results on closeness between solutions of the difference equation (3) and the difference-differential equation (1) for small $\mu$, we can easily obtain an analog of Lemma $G$ for the delay differential equation (1).

THEOREM 5. For any triple $(p, q, d)$ and arbitrary $\varepsilon>0$ and $\delta>0$, there exist a parameter set $\Omega_{p, q, d}^{*} \subset \Omega_{p, q, d}^{0}$ with mes $\left(\Omega_{p, q, d}^{0} \backslash \Omega_{p, q, d}^{*}\right)<\delta$, and also $T>T_{0}+q+2 d$, an integer $k>0$, and $\mu_{0}>0$ such that for any $\mu<\mu_{0}$, for any $\omega \in \Omega_{p, q, d}^{*}$, and for any initial function $\psi \in P L$, the solution $x_{\varphi}^{\mu}$ of the delay differential equation (1) is an $\varepsilon$-periodic function of type $(p, q, d)$ on the interval $\left[T_{0}, T\right]$.

Theorem 6 below follows from Theorem 5 and properties of the bifurcation diagram for the difference equation (3). It describes the bifurcation diagram for equation (1) and is an analog of Theorem 3 for this case. 
THEOREM 6. (1) Let $\omega_{i} \in \Omega_{p_{i}, q_{i}, d_{i}}^{0}, i=1,2$, be two collections of parameters and $p_{1} / q_{1}<p_{2} / q_{2}$. Let $N$ be arbitrary integer $>1$ and $\Im \subset \Omega$ be an arc connecting $\omega_{1}$ and $\omega_{2}$ such that $\Im \cap \Omega_{p, q, d} \neq \emptyset$ implies $q+2 d \leqslant N$ and $\Im \cap \Omega_{p, q, d}^{0} \neq \emptyset$. Then for any $\varphi \in P L, T>T_{0}+N, \varepsilon>0$, there exists $\mu_{0}>0$ such that for any $p, q$ with $p_{1} / q_{1} \leqslant p / q \leqslant p_{2} / q_{2}$, there is a collection of parameters $\omega \in \Im$ for which the solution $x_{\varphi}^{\mu}$ of equation (1) with any $\mu<\mu_{0}$ is an $\varepsilon$-periodic function of type $(p, q, d)$ on the interval $\left[T_{0}, T\right]$ with some $d, q+2 d \leqslant N$.

(2) Let $\omega_{i} \in \Omega_{p, q, d_{i}}^{0}, i=1,2$, be two collections of parameters and $d_{1}<d_{2}$. Let $\Im \subset \bigcup_{d=d_{1}}^{d_{2}} \Omega_{p, q, d}$ be an arc connecting $\omega_{1}$ and $\omega_{2}$ such that $\Im \cap \Omega_{p, q, d}^{0} \neq \emptyset$ for $d_{1} \leqslant d \leqslant d_{2}$. Then for any $d, d_{1} \leqslant d \leqslant d_{2}$, any $\psi \in P L$, any $T>T_{0}+q+2 d$, and any $\varepsilon>0$, there exist $\mu_{0}>0$ and a collection of parameters $\omega \in \Im$ for which the solution $x_{\varphi}^{\mu}$ of equation (1) with any $\mu<\mu_{0}$ is an $\varepsilon$-periodic function of type $(p, q, d)$ on the interval $\left[T_{0}, T\right]$.

\section{REFERENCES}

[1] K.L. Cooke and D. Krumme, 'Differential difference equations and nonlinear initial-boundary-value problems for linear hyperbolic partial differential equations', J. Math. Anal. Appl. 24 (1968), 372-387.

[2] A.D. Fedorenko, V.V. Fedorenko, A.F. Ivanov and A.N. Sharkovsky, 'Farey tree for stable periodic waves in a transmission line', (CADSEM Report 96-008, Deakin University, 1996).

[3] E.V. Gaushus, Investigation of dynamical systems using the point mapping method, (in Russian) (Nauka, Moscow, 1976).

[4] A.F. Ivanov and A.N. Sharkovsky, 'Oscillations in singularly perturbed delay equations', Dynam. Report. 1 (1991), 165-224.

[5] J. Nagumo and M. Shimura, 'Self-oscillations in a transmission line with a tunnel diode', Proc. IRE 49 (1961), 1281-1291.

[6] A.N. Sharkovsky, Yu.L. Maistrenko and E.Yu. Romanenko, Difference equations and their applications, Mathematics and Its Applications 250 (Kluwer Academic Publishers, Dordrecht, Boston, London, 1993).

[7] A.N. Sharkovsky, 'Chaos from a time-delayed Chua's circuit', IEEE Trans. Circuits and Systems I 40 (1993), 781-783.

[8] A.N. Sharkovsky, 'Ideal turbulence in an idealized time-delayed Chua's circuit', Internat. J. Bifur. Chaos Appl. Sci. Engrg. 4 (1994), 303-309.

[9] O.M. Sharkovsky, A.F. Ivanov, V.V. Fedorenko and O.D. Fedorenko, 'Coexistence of periodic orbits for a class of discontinuous maps', (in Ukrainian), Proc. Nat. Acad. Sci. Ukraine 11 (1996), 20-25.

[10] A.A. Witt, 'On the theory of the violin string', (in Russian), J. Tech. Phys. 6 (1936), 1459-1470. 
Institute of Mathematics

National Academy of Sciences of Ukraine

Kive

Ukraine

CADSEM and School of

Computing and Mathematics

Deakin University

Melbourne Vic

Australia.

and

Institute of Mathematics

National Academy of Sciences of Ukraine

Kive

Ukraine
Institute of Mathematics

National Academy of Sciences of Ukraine

Kive

Ukraine

Institute of Mathematics

National Academy of Sciences of Ukraine

Kive

Ukraine 